\title{
Plasma intestinal alkaline phosphatase isoenzymes in neonates with bowel necrosis
}

\author{
R McLachlan, J Coakley, L Murton ${ }^{\star}$, N Campbell
}

\begin{abstract}
Aim-To determine if the intestinal isoenzymes of alkaline phosphatase (ALP) are biochemical markers of bowel necrosis in neonates.

Methods-Plasma ALP isoenzymes were measured in 22 babies with bowel necrosis, histologically confirmed, and in 22 matched controls. The isoenzymes were also measured in 16 infants with signs of necrotising enterocolitis, who recovered without histological confirmation of bowel necrosis. The isoenzymes were separated by polyacrylamide gel electrophoresis. Auxiliary tests for identification included neuraminidase digestion and treatment with monoclonal and polyclonal antiplacental antibodies.
\end{abstract}

Results-Intestinal ALP was detected in 16 infants with bowel necrosis-13 had fetal intestinal ALP (FI-ALP) and three had adult intestinal ALP (AI-ALP). FIALP was detected in nine of the controls. In the babies with bowel necrosis intestinal ALP was found over all gestations, but in the controls only in those less than 34 weeks. The percentages of total ALP activity due to intestinal ALP were significantly higher in those with bowel necrosis compared with matched controls $(p=0.028)$. In babies of all gestations diagnostic sensitivity for the presence of intestinal ALP as a marker of bowel necrosis was $73 \%$ and diagnostic specificity $59 \%$. In babies greater than 34 weeks' gestation, diagnostic sensitivity fell to $60 \%$ but the test became completely specific. In two babies FI-ALP increased from zero/trace to high activity coincident with the episode of bowel necrosis. In 16 babies with signs of necrotising enterocolitis but unconfirmed bowel necrosis FI-ALP was detected in four.

Pathology

Department, Peter MacCallum Cancer Institute, Melbourne, Victoria, Australia

R McLachlan

Department of Clinical Biochemistry, Royal Children's Hospital, Parkville, Victoria, Australia L Murton

N Campbell

J Coakley

^Deceased

Correspondence to: Dr J Coakley Department of Biochemistry The Children's Hospital Camperdown NSW Australia 2050

Accepted for publication

17 February 1993 enortity. Necrot enterocolitis is the most common cause. sents with abdominal distension, bilious vomiting, and bloody stools. Pneumatosis intestinalis, with or without intrahepatic gas, is the distinctive radiological feature. Necrotising
Bowel necrosis in newborns causes consider- enterocolitis is the most common acquired intestinal emergency in the neonatal intensive care unit. ${ }^{1}$

The symptoms and signs of necrotising enterocolitis may be non-specific, resembling other forms of sepsis, and diagnosis in the initial stages of the illness may be difficult. There are no specific tests for the condition, apart from radiography for pneumatosis intestinalis or intrahepatic gas, and even these signs may be difficult to interpret. ${ }^{2}$ In addition, necrotising enterocolitis can be present in the absence of intramural intestinal gas. Therefore, additional diagnostic tests have been sought, including measurement of the intestinal enzymes, acid phosphatase, ${ }^{3}$ and hexosaminidase. ${ }^{4}$

The purpose of this study was to evaluate the measurement of isoenzymes of alkaline phosphatase (ALP) as diagnostic markers of bowel necrosis in the newborn. The isoenzymes of ALP have been subdivided into four classes determined by the primary structure of their peptide units. ${ }^{5}$ Class 1 contains the common or tissue non-specific ALP and includes the liver and bone types, class 2 contains AI-ALP, class 3 contains placental ALP and placental-like variants and class 4 contains FI-ALP. FI-ALP is believed by some to be a heterodimer composed of placental and AI-ALP. ${ }^{6}$ Separate genes code for the first three classes, but it is uncertain if this is the case for FI-ALP. ${ }^{7}$ Unlike AI-ALP, there does not seem to be any relation between FIALP and blood group states. 9 AI-ALP and FI-ALP are found in the cell membranes of the bowel mucosa and may be released into the circulation in increased amounts when ischaemia or infarction occurs. Previous studies have shown that the changeover from the synthesis of fetal to adult intestinal enzyme begins at about 28 to 32 weeks' gestation. ${ }^{10}$ In 1983 , one of us (RMcL) first observed circulating FI-ALP in a 4 week old, 26 week gestation infant. Circulating FI-ALP has since been found mainly in premature babies of less than 35 weeks' gestation in the absence of bowel pathology, ${ }^{11}$ and has been studied in relation to the introduction of milk feeding. ${ }^{9}$ In this last investigation, it was noted that four term neonates who developed necrotising enterocolitis had significantly higher concentrations of plasma FI-ALP on the first day of life compared with other term infants. The authors cited this as indirect evidence that increased intestinal permeability may lead to greater activity of FI-ALP in the circulation and suggested a prospective study to evaluate the measurement of FI-ALP in necrotising enterocolitis. 
Our study of plasma ALP isoenzymes was directed particularly towards infants with necrotising enterocolitis, but we also included a small number of babies with other conditions associated with bowel necrosis.

\section{Methods}

Our principal study group consisted of babies with bowel necrosis which had been histologically confirmed either following surgery or at post mortem examination. This group comprised 22 infants (16 male) whose gestations ranged from 25 to 40 weeks (median 34 weeks). Nineteen babies had bowel necrosis following necrotising enterocolitis and three developed the condition as a result of volvulus of the intestine. Two babies died without undergoing surgery, the rest had bowel resection operations. Each baby with bowel necrosis was matched to a control infant of similar gestation, who did not have any clinical evidence of bowel pathology. In the control group there were 12 males and gestations ranged from 24 to 40 weeks (median 33 weeks).

The samples for study of ALP isoenzymes were obtained close to the time of suspected bowel necrosis and usually when blood was being taken for other tests. Of the 20 babies undergoing surgery, 16 had blood taken either before or on the day of surgery and four babies had samples taken the next day. Some babies had multiple samples taken both before and after operation. In four infants we were able to obtain leftover plasma before clinical signs of necrotising enterocolitis had become apparent. In general, the sample closest to the onset of the episode of bowel necrosis was selected for comparison with a control. Control blood samples and samples from the babies with bowel necrosis were matched closely in terms of postnatal age at time of collection. The samples from the controls were taken between one and 42 days after birth (median 10 days), while the corresponding bowel necrosis samples were taken between one and 44 days (median 11 days).

In addition to the babies with confirmed bowel infarction, we also received samples from 16 babies with possible/probable necrotising enterocolitis, who were treated conservatively and recovered without the need for surgery. The symptoms in these babies varied widely - some had only minimal symptoms of necrotising enterocolitis; others had the complete clinical picture. Gestations in this group ranged from 24 to 42 weeks (median 37 weeks). Only five of the babies were less than 35 weeks' gestation. Because the diagnosis was uncertain in some of these babies and because we did not have definite histological evidence of bowel infarction in them, we excluded them from the principal study group. Nevertheless, some of their findings for plasma ALP isoenzymes are of interest and so have been included in the results.

Approval for this study was obtained from the Ethics Committees of the Royal Children's and Royal Women's Hospitals,

\section{Melbourne.}

Total ALP activity was assayed using a Kodak Ektachem 700 analyser (Eastman Kodak, Rochester, New York). The isoenzyme analysis was performed by anionic disc gel electrophoresis in thin vertical polyacrylamide gels using a discontinuous buffer system as previously described. ${ }^{12}$ The isoenzymes were concentrated (stacked) and then separated according to both electrical charge and molecular size. They were detected by their enzymatic activity with the chromogen, nitro blue tetrazolium, and the substrate 5-bromo-4-chloro-3-indolylphosphate. Auxiliary tests used for the identification of FI-ALP (performed before electrophoresis) included neuraminidase digestion $^{13}$ and treatment with monoclonal and polyclonal antiplacental antibodies. ${ }^{14}$ The proportion of each isoenzyme was measured by densitometry and expressed as a percentage of the total ALP activity.

Total ALP activity, percentage intestinal ALP, and absolute intestinal ALP in the bowel necrosis babies were compared with those in the matched controls using the Wilcoxon matched pairs signed rank sum test. The Minitab (version 8) computer program was used to carry out the statistical analysis.

\section{Results}

There was a wide scatter in total ALP activity, both in the babies with bowel necrosis and in the controls. In the former group ALP activity ranged between 53 and $1123 \mathrm{IU} / \mathrm{l}$, while in the matched control group ALP activity ranged between 28 and $567 \mathrm{IU} / \mathrm{l}$. There was no significant difference between the matched pairs for total ALP activity $(\mathrm{p}=0.897)$.

Typical patterns of ALP isoenzymes after polyacrylamide gel electrophoresis are shown in fig 1. FI-ALP appears as a wide band because of its variable sialic acid content. In babies the bone ALP band is also broader than its adult counterpart. The mobility changes effected by pre-treatment of isoenzymes with neuraminidase, which removes sialic acid residues, are shown. The reduction in electrophoretic heterogeneity of both FIALP and bone ALP, but not AI-ALP, is evident. The use of antiplacental antibodies as an aid to the identification of the various isoenzymes is also illustrated. The antibodies bind to placental, AI-ALP, and FI-ALP; the consequent size increase of the complexes prevents them from entering the separating gel.

In keeping with previous studies, ${ }^{11}$ bone ALP was found to be the major isoenzyme present in the babies' plasma, except for those infants who had greater than $50 \%$ FIALP in association with their episodes of bowel necrosis. The liver isoenzyme was found in 13 babies (both bowel necrosis group and controls) and generally in small amounts. Seven of these 13 infants were equal to or greater than 34 weeks' gestation. 


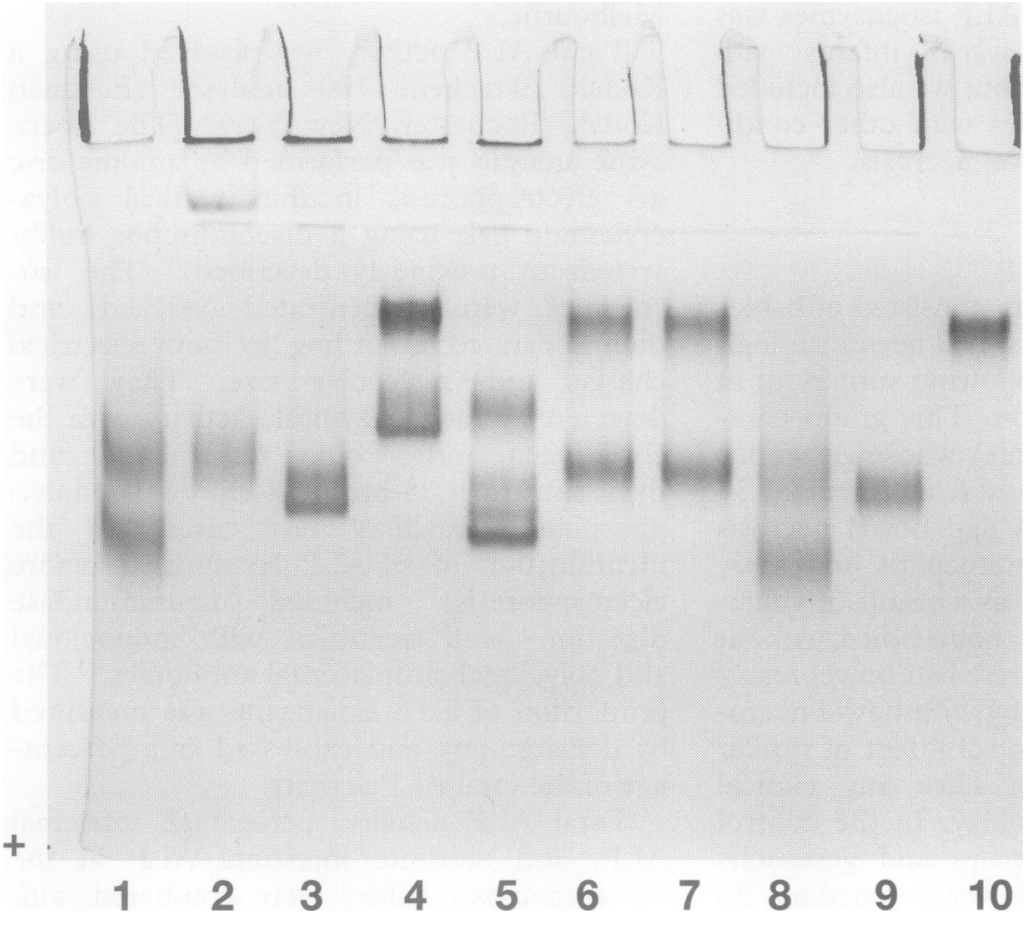

Figure 1 Polyacrylamide gel electrophoresis of ALP isoenzymes. Anode (+) is indicated on the figure.

Tracks 1 and 8: neonatal serum containing FI-ALP (anodal band) and bone ALP.

Track 2: serum shown in track 1 treated with polyclonal antiplacental antibody.

Track 3: reference sample (see track 5) treated with polyclonal antiplacental antibody.

Track 4: reference sample treated with neuraminidase. Isoenzymes, from the anode, are

placental, adult intestinal, liver/bone.

Track 5: reference sample containing, from the anode, placental, liver, bone and adult intestinal ALPs.

Tracks 6 and 7: serum shown in track 1, treated with neuraminidase. Isoenzymes are, from the anode, FI-ALP and bone.

Track 9: bone ALP (adult).

Track 10: bone ALP (adult) treated with neuraminidase.

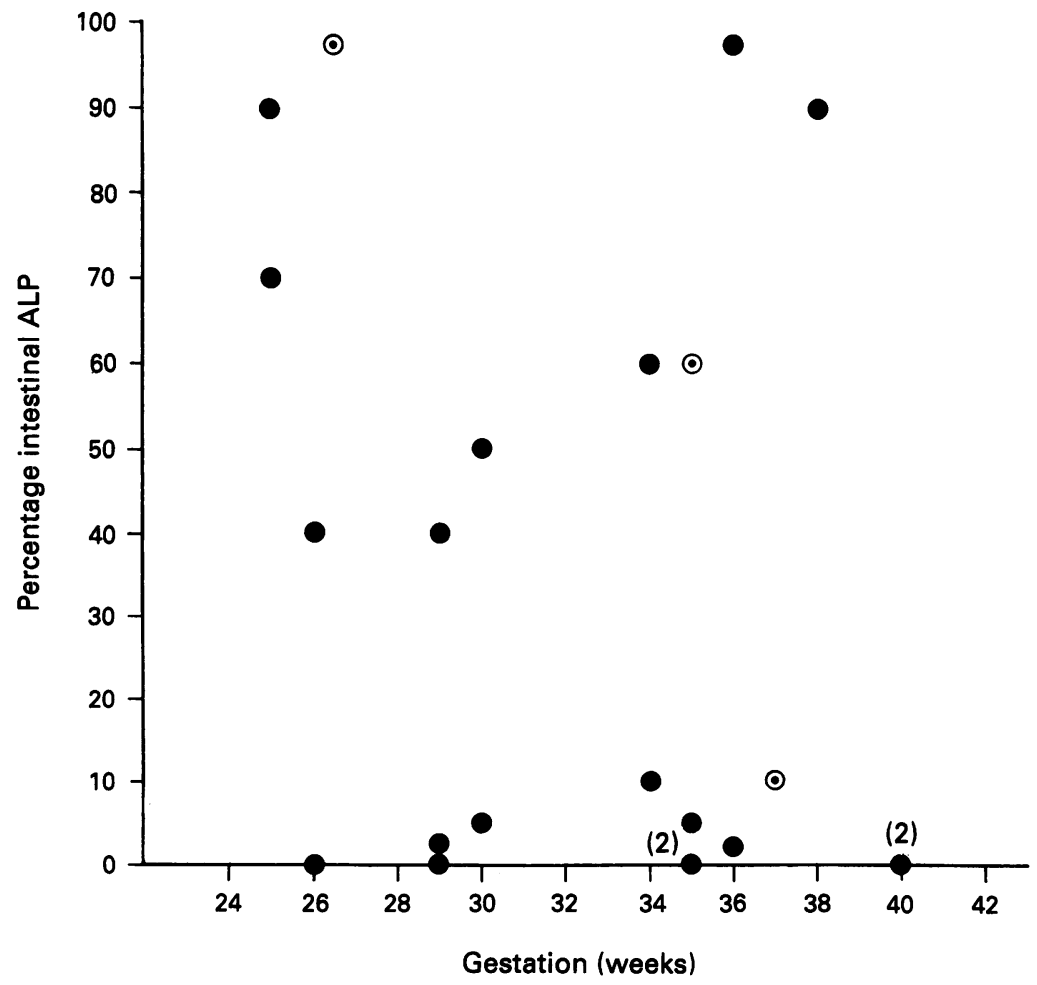

Figure 2 Percentage intestinal ALP compared with gestation in bowel necrosis group. Figure ${ }^{\text {FI-ALP; } \odot=A I-A L P . ~ N u m b e r s ~ i n d i c a t e ~ m u l t i p l e ~ r e s u l t s ~ a t ~ t h e ~ o n e ~ l o c u s . ~ " T r a c e " ~}$ values recorded at $2 \cdot 5 \%$.
In figs 2 and 3 percentage plasma intestinal ALP activity in the babies with bowel necrosis and in the controls is plotted against gestational age. Figures 4 and 5 show the lack of association between postnatal age and the presence of intestinal ALP. Of the infants with bowel necrosis who had detectable intestinal ALP activity, 13 had FI-ALP, two had AI-ALP and a third baby (described in more detail below) most likely had a variant of AI-ALP. The gestations of the three babies with AI-ALP were 27, 35, and 37 weeks' respectively. There was no detectable intestinal ALP activity in six of the 22 infants with confirmed bowel necrosis, including two babies in whom the samples were taken preoperatively. Of the controls, nine had intestinal ALP (all had FI-ALP and all were less than 34 weeks' gestation). In the other 13 controls no intestinal ALP was found. In addition, eight of the babies with bowel necrosis had greater than $50 \%$ intestinal ALP; none of the controls had a value greater than $50 \%$. The percentage of intestinal ALP was significantly higher in those with bowel necrosis compared with the matched controls $(p=0.028)$. The median of the differences between the matched pairs was $20 \%$, with a $95 \%$ confidence interval of 1.3 to $32.5 \%$. In the case of absolute intestinal ALP, however, the difference in results between the patients and the controls was not significant $(p=$ 0.095). The median of the differences between the matched pairs for absolute intestinal ALP was $24 \mathrm{IU} / 1$, with the patients higher than the controls, but the $95 \%$ confidence interval ranged from -10 to $205 \mathrm{IU} / 1$.

Specific results in five of the babies are of particular interest. In one infant of 25 weeks' gestation a blood sample taken at 15 days of age had a total ALP activity of $716 \mathrm{IU} / 1$ $(>95 \%$ bone ALP) and at this time there were no symptoms of necrotising enterocolitis. About three days later, the baby developed abdominal distension, erythema, and tenderness. A blood sample taken after the onset of these signs had a total ALP activity of $881 \mathrm{IU} / 1$ (70\% FI-ALP). Surgery revealed the presence of necrotising enterocolitis and the baby died soon afterwards. Similarly, another baby of 26 weeks' gestation had only a trace of FI-ALP when there were no symptoms of necrotising enterocolitis, but $60 \%$ FIALP on the day of surgery for bowel necrosis. Another infant of 37 weeks' gestation had no definite signs of necrotising enterocolitis at 33 days of age when the first blood sample was assayed for ALP isoenzymes. The result was a total ALP of $272 \mathrm{IU} / 1$ ( $>95 \%$ bone ALP). The next day, an abdominal radiograph showed intramural gas and a blood sample on the day following radiography had a total ALP activity of only $81 \mathrm{IU} / 1$, with $5 \%$ liver, $85 \%$ bone, and $10 \%$ AI-ALP. At surgery infarcted bowel was present. About two days later, total ALP had risen to $342 \mathrm{IU} / 1$ with $>95 \%$ liver/bone ALP and just a trace of AIALP. The baby died the next day. Another premature baby of 30 weeks' gestation had a trace of FI-ALP when there were no symp- 


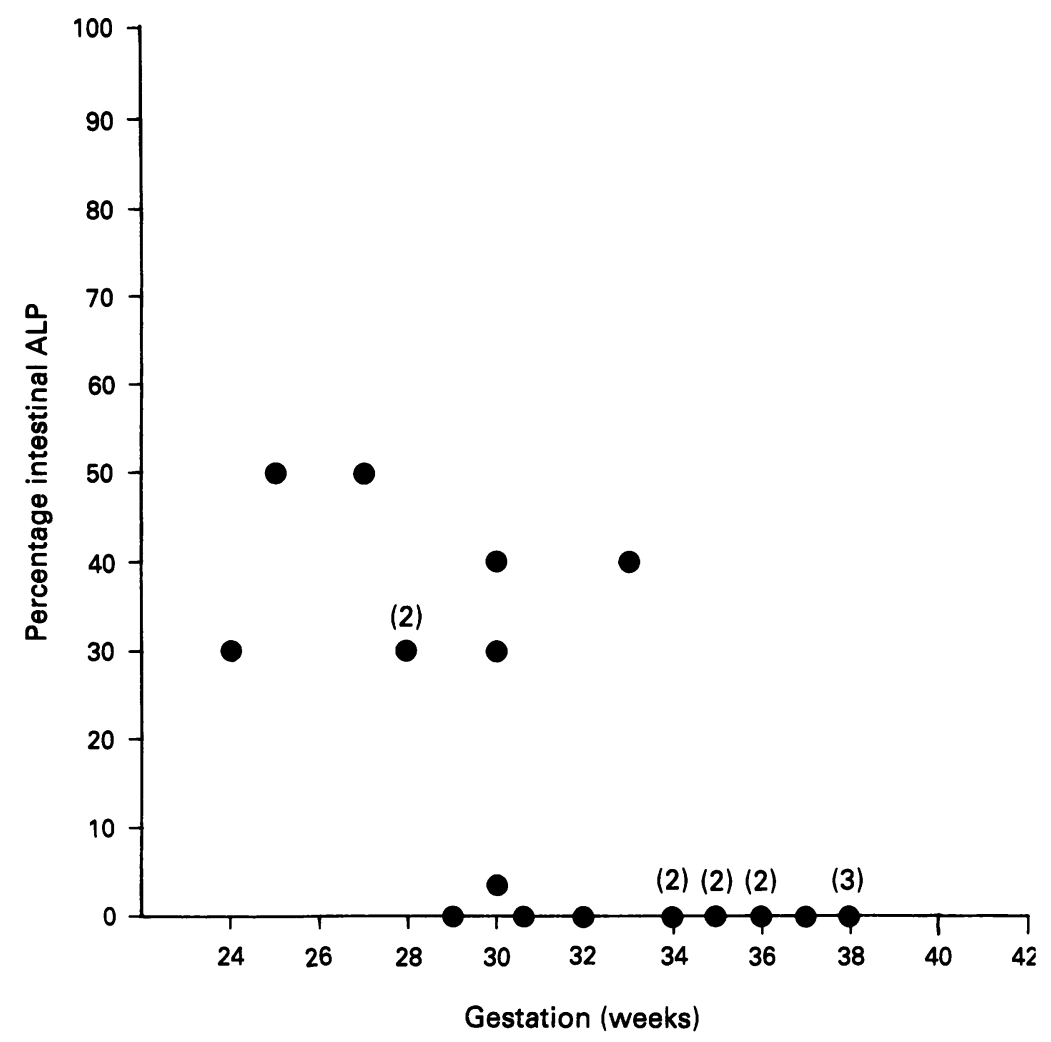

Figure 3 Percentage intestinal ALP compared with gestation in control group. All babies with positive results had FI-ALP. Numbers indicate multiple results at the one locus. "Trace" values recorded at $2 \cdot 5 \%$.

toms of necrotising enterocolitis. The next day the percentage FI-ALP had risen to $5 \%$ and two days after this the baby had signs of necrotising enterocolitis. At surgery, extensive infarction of the bowel was present. The percentage FI-ALP remained at $5 \%$ for five days

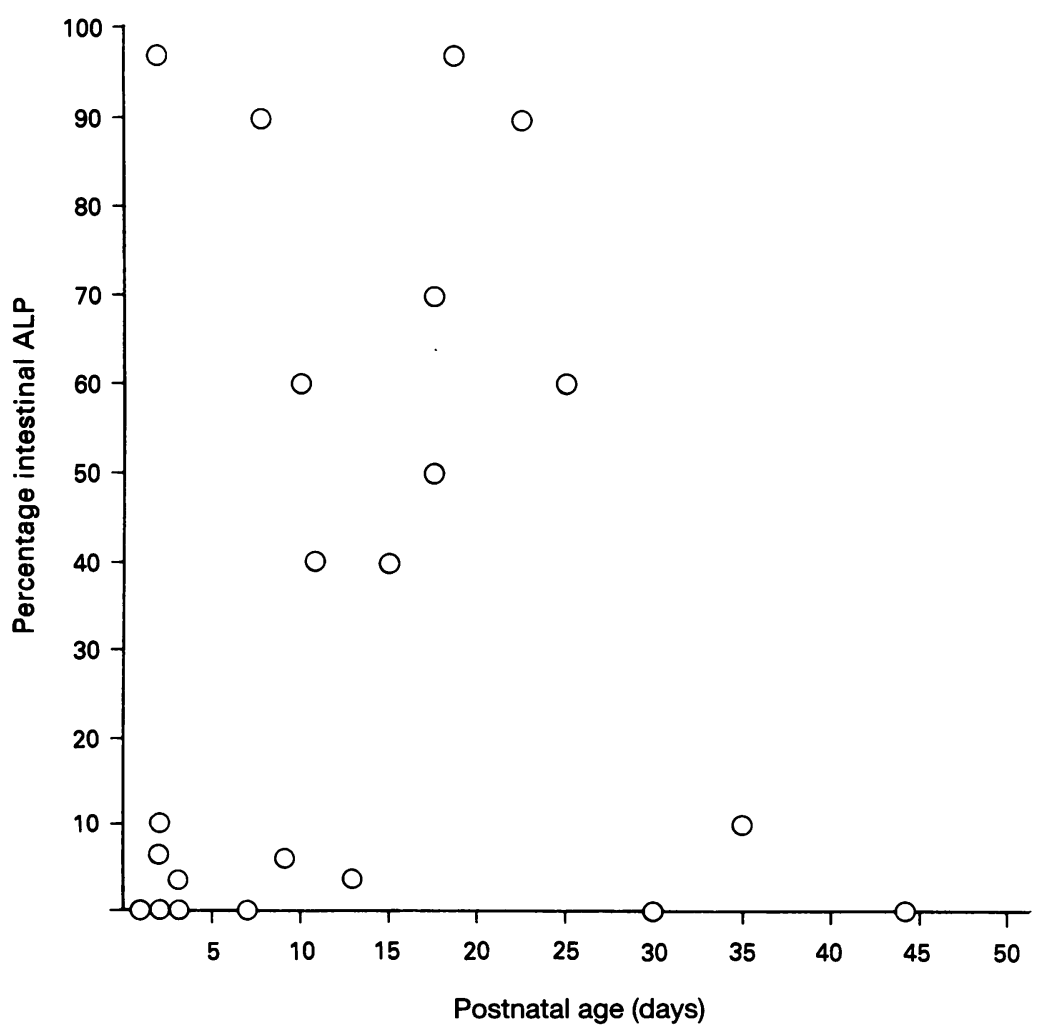

Figure 4 Percentage intestinal ALP compared with postnatal age in bowel necrosis group. before dropping back to trace levels on the day before the baby died. The fifth baby, of 35 weeks' gestation, had multiple problems, including severe haemorrhage, and developed necrotising enterocolitis as a pre-terminal event. The isoenzyme pattern in this infant was not typical-a band migrating in the region of AI-ALP was partially sensitive to neuraminidase but reacted with antiplacental antibody. This isoenzyme had the characteristics of "variant" intestinal ALP. ${ }^{15}$ Its percentage of total ALP rose from 20 to $60 \%$ over a period of 10 days, and about three days after the last ALP measurement, the baby died.

Intestinal ALP was detected in only four out of the 16 babies with possible/probable necrotising enterocolitis for whom no histological diagnosis of bowel necrosis was available. The data on these four babies are summarised as follows. The first was born at 40 weeks' gestation, had intramural bowel gas, and had variable FI-ALP activity in two blood samples taken on the same day. The first sample contained no FI-ALP activity, the second obtained about 15 hours later had $30 \%$ atypical FI-ALP-atypical because its mobility was intermediate between placental and bone isoenzymes and was unaffected by neuraminidase treatment. A third sample, collected five days later, did not contain intestinal ALP. The second baby of 28 weeks' gestation presented with rectal bleeding and abdominal distension but his main problems were anaemia and sepsis. Ten per cent FIALP was detected in this baby's plasma, decreasing to a trace over three days. The third infant, born at 35 weeks, had minimal symptoms of necrotising enterocolitis-mainly abdominal distension. This baby had $20 \%$ FI-ALP. The final baby, of 36 weeks, had intrauterine growth retardation, primary hypothyroidism, abdominal distension, malaena and bile stained vomiting. Fifty per cent FI-ALP was detected in this baby's plasma. Intestinal ALP was not detected in the remaining 12 babies. This group included one infant of 42 weeks' gestation who had intramural bowel gas, rectal bleeding, and abdominal distension. This baby later developed a stricture of the distal colon.

Tests of diagnostic sensitivity and specificity $^{16}$ were carried out on the 22 babies with confirmed bowel necrosis and the 22 controls. The sensitivity for the presence of intestinal ALP as a marker of bowel necrosis was $73 \%$, specificity $59 \%$, and the predictive value of a positive test was $64 \%$. The overall efficiency of the test-the percentage of patients correctly classified as having bowel necrosis or not-was $66 \%$. We then applied these tests just to the babies greater than 34 weeks' gestation (10 with bowel necrosis and eight controls) because the more premature babies were more likely to have higher FIALP values irrespective of the presence or absence of bowel pathology. ${ }^{11}$ Diagnostic sensitivity fell slightly to $60 \%$ but specificity and the predictive value of a positive result rose to $100 \%$. The efficiency of the test in the more mature babies was $70 \%$. 


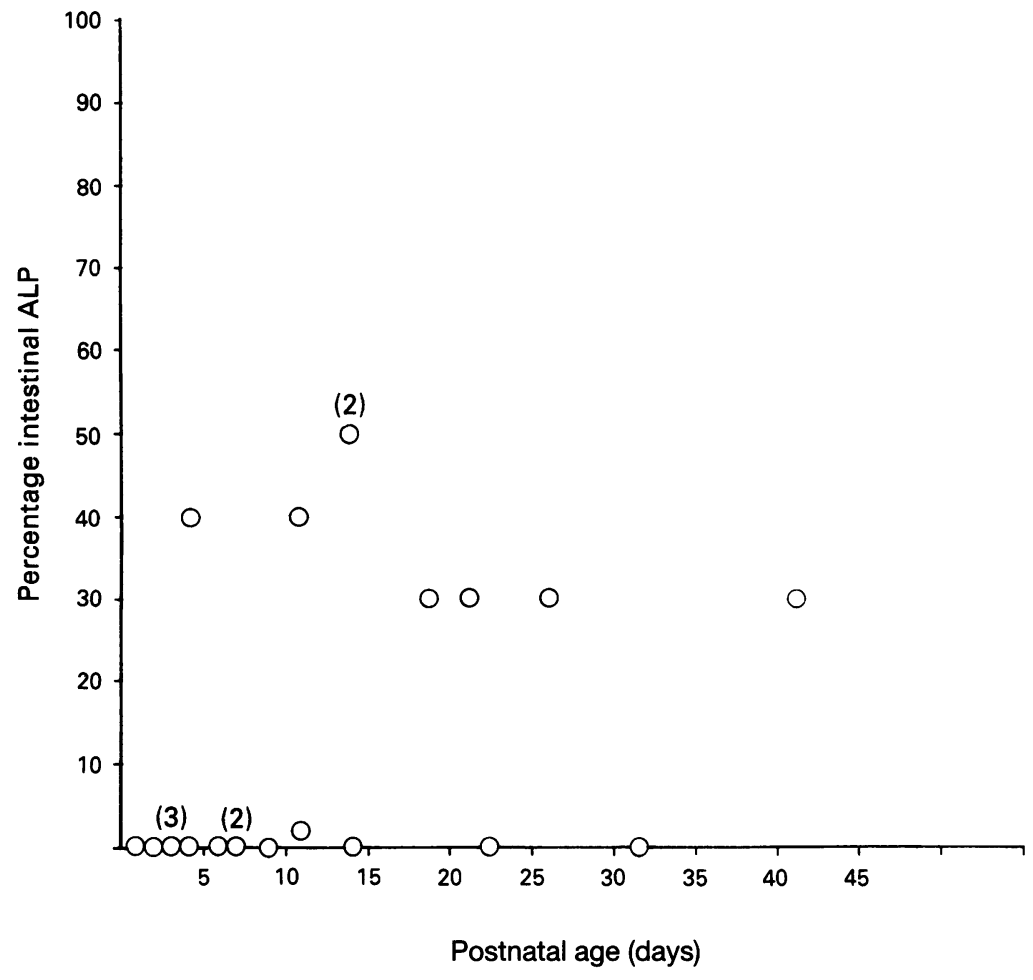

Figure 5 Percentage intestinal ALP compared with postnatal age in control group. Numbers indicate multiple results at the one locus.

\section{Discussion}

In this investigation we found plasma intestinal ALP activity in 16 out of 22 babies with confirmed bowel necrosis but in only nine out of 22 matched controls who had no clinical evidence of bowel pathology. In the bowel necrosis group the presence of intestinal ALP was independent of gestational age, but in the controls it was confined to those less than 34 weeks' gestation. Most of the intestinal ALP was fetal in origin but a small number of babies with bowel necrosis had the adult form of the isoenzyme. In some babies we obtained evidence of a changing isoenzyme pattern which suggested that intestinal ALP was released into the circulation coincident with the episode of bowel infarction. In one baby who had extramural intestinal gas but did not undergo operation an apparent variant form of FI-ALP was detected. Its electrophoretic mobility was reduced and was unaffected by neuraminidase, indicating that it did not contain sialic acid. Crofton and Hume ${ }^{9}$ noted decreased mobility of FI-ALP isolated from meconium and stool, but not serum, during the first week after birth, but did not state whether this form of FI-ALP was neuraminidase sensitive.

One of the factors leading to the presence of FI-ALP in the plasma may be the introduction of milk feeding which has been shown to induce activity of the isoenzyme. Premature babies have a much higher and more prolonged increase in circulating FIALP with milk feeds. In term infants the increase is much smaller and the bone isoenzyme remains the predominant form of circulating ALP. ${ }^{9}$ For many of the babies in this study, particularly those of less than 35 weeks' gestation, we are therefore unable to conclude that the appearance of intestinal ALP definitely coincided with the episode of bowel necrosis. Nevertheless, the very high FI-ALP activity found in some babies suggests added release of this isoenzyme into the circulation.

In the 16 babies who survived without surgery, only four had detectable FI-ALP in their plasma. None had AI-ALP. Three of the four were of 35 weeks' gestation or more. Therefore, intestinal ALP may be released into the circulation in some babies with less severe forms of bowel inflammation but more investigations would be needed to prove this.

Tests of diagnostic sensitivity and specificity were confined to the group with confirmed bowel necrosis and the controls. The 16 infants referred to above were omitted from this analysis because they did not have a definite histological diagnosis of bowel necrosis. Diagnostic sensitivity and specificity varied according to gestation. For the group as a whole, sensitivity for the presence of intestinal ALP as a marker of bowel necrosis was $73 \%$, specificity $59 \%$, and the predictive value of a positive test $64 \%$. For babies greater than 34 weeks' gestation the test was less sensitive $(60 \%)$ but completely specific. The latter finding occurred because we did not detect intestinal ALP in our eight control infants who were greater than 34 weeks' gestation. This is only a small number of subjects, however, and FI-ALP has been reported to occur occasionally in babies of such gestation. ${ }^{9}$ In addition, four infants with definite bowel necrosis in the more mature group did not have measurable concentrations of intestinal ALP in their plasma.

We conclude that the detection of plasma intestinal ALP is not a reliable biochemical marker of bowel necrosis in the newborn because of the false positive and false negative results obtained. Nevertheless, intestinal ALP seems to be released into the circulation in some babies following bowel ischaemia/ infarction. It is also possible that, because of the design of this study, short-lived transient rises in intestinal ALP in some of the babies were missed. Further study may lead to the finding of other bowel constituents which are more sensitive and specific markers for bowel necrosis.

Kliegman R M, Fanaroff A A. Necrotizing enterocolitis. NEngl F Med 1984;310:1093-103.

2 Mata A G, Rosengart R M. Interobserver variability in the radiographic diagnosis of necrotizing enterocolitis. Pediatrics 1980;66:68-71.

3 Takieddine F N, Marchant C, Dahms B, Kwong M, King $\mathrm{K}$. Plasma acid phosphatase in necrotizing enterocolitis. Pediatr Res 1980;14:613.

4 Lobe T M, Richardson C J, Rassin D K, Mills R, Schwartz M. Hexosaminidase: a biochemical marker for necrotizing enterocolitis in the preterm infant. $\mathrm{Am} \mathcal{F}$ Surg 1984;147:49-51.

5 Stigbrand $T$. Introduction and nomenclature. In Stigbrand $\mathrm{T}$, Fishman $\mathrm{W} H$, eds. Human alkaline phosStigbrand T, Fishman W $\mathrm{H}$, eds. Human alkaline phosBehrens C M, Enns C A, Sussman H H. Characterization Behrens C M, Enns C A, Sussman H H. Characterization
of human foctal intestinal alkaline phosphatase. Biochem f 1983;211:553-8.

7 Millan J L. Oncodevelopmental expression and structure of alkaline phosphatase genes. Anticancer Res 1988;8 995-1004

8 Henthorn P S, Raducha M, Kadesh T, Weiss M J, Harris 
H. Sequence and characterization of the human intestinal alkaline phosphatase gene. F Biol Chem 1988;263: 12011-19.

9 Crofton P M, Hume R. Alkaline phosphatase isoenzymes in the plasma of preterm and term infants: serial measurements and clinical correlations. Clin Chem 1987;33. 1783-7.

10 Mulivor R A, Hannig V L, Harris H. Developmental change in human intestinal alkaline phosphatase. Proc Natl Acad Sci USA 1978;75:3909-12.

11 Crofton P M. Properties of alkaline phosphatase isoenzymes in plasma of preterm and term neonates. Clin

12 McLachlan R, Biegler B, Raines G, Sykes S. Alkaline phosphatase isoenzymes: techniques. In: Biegler B, Sykes S, eds. The clinical biochemist monograph: Advanced electrophoretic techniques in clinical diagnosis. Sydney: Australian Association of Clinical Biochemists, 1989:18-21.

13 Robinson C J, Pierce J E. Differential action of neuraminidase on human serum alkaline phosphatases. Nature 1964;204:472-3.

14 Sussman H, Small P A, Cotlove E. Human alkaline phosphatase. Immunochemical identification of organphosphatase. Immunochemical identification of organ

15 Panteghini M. Benign inherited hyperphosphatasemia of intestinal origin: report of two cases and a brief review of the literature. Clin Chem 1991;37:1449-52.

16 Galen R S, Peters T. Analytical goals and clinical relevance of laboratory procedures. In: Tietz $\mathrm{N}$, ed. Textbook of clinical chemistry. Philadelphia: W B Saunders Company, 1986:387-409. 